\title{
Verdad, racionalidad, universidad. Perspectivas sobre la universidad y la teología en la actualidad
}

\author{
Truth, Rationality, University: Today's Perspectives About \\ University and Theology
}

\section{Cyrille MiCHON}

Université de Nantes. Centre Atlantique de Philosophie Nantes. Francia

cyrille.michon@univ-nantes.fr

Resumen: La enseñanza de la teología en la universidad se plantea a menudo como un problema, y sin embargo, históricamente fue considerada la disciplina más alta. Parece que uno de los principales motivos de esta situación es el conflicto que algunos ven entre razón y autoridad (1). El papa Benedicto XVI ha presentado a la vez un diagnóstico y un remedio a la «crisis de la universidad», desarrollando de diversas maneras la idea de una «racionalidad ampliada» (2). Por esta vía, la universidad secular podría abrir un espacio a la enseñanza de la teología manteniendo al mismo tiempo su exigencia de racionalidad, incluso si la naturaleza de esta enseñanza pudiera adoptar una forma distinta a la de la teología que se imparte en las universidades confesionales (3).

Palabras clave: Verdad, Racionalidad, Teología en la universidad.
Abstract: The university teaching of theology, which was historically considered as the highest discipline, is often times deemed a problem. It seems that one of the principal causes of this state of affairs is the conflict, conceived by some thinkers, between reason and authority. Pope Benedict XVI offers both a diagnosis and a remedy to the «crisis of university» by developing the idea of «extended rationality». Through this idea, secular universities could, without compromising its demand of rationality, provide room for theology, even though it does not adopt the way theology is imparted in confessional universities.

Keywords: Truth, Rationality, Theology in University. 


\section{1. ¿PUEDE La UNIVERSIDAD DEJAR ESPACIO A UNA AUTORIDAD QUE NO SEA LA RAZÓN?}

$\mathrm{L}$ a universidad es la institución dedicada a la investigación y a la enseñanza. Es laboratorio y escuela. Su objeto es el conocimiento y, por tanto, la verdad, para descubrirla y trasmitirla. Pero no se trata de cualquier verdad, sino de la que es relevante para la racionalidad: la verdad cuya búsqueda y enseñanza es racional, argumentada, «científica» por tanto, en un sentido amplio que incluye tanto las ciencias positivas como las humanidades: derecho, letras y las llamadas ciencias humanas. Estas disciplinas encuentran en la universidad un lugar por derecho propio frente al arte o a los discursos políticos o religiosos. $\mathrm{O}$ al menos frente a los discursos religiosos de tipo parenético, es decir, la predicación bajo una u otra forma. Aunque este tipo de discursos haya podido tener un espacio en la universidad (pensemos en los sermones universitarios de Santo Tomás o de J. H. Newman), o todavía puedan ocuparlo, ese contexto no constituye su razón de ser, ya que su sitio propio se encuentra en los lugares de culto. Sin embargo, el discurso «teológico», la profundización por medio de la razón humana en el contenido de una doctrina religiosa, puede aspirar a formar parte de esa búsqueda racional de la verdad y de su enseñanza. Es más, la teología ha presidido la institución universitaria durante mucho tiempo como reina de las ciencias. Hoy ya no es así: numerosas universidades no dejan hueco alguno a la teología; y las que lo hacen, que a menudo son universidades confesionales, no le otorgan claramente el estatus privilegiado, propio de la sabiduría, que le daban los Medievales.

Este cambio podría explicarse simplemente por el papel predominante de la Iglesia en la universidad medieval y su progresiva retirada institucional de las universidades modernas (retirada total en las universidades públicas, y relativa, en diferentes grados, en las universidades confesionales). Y podríamos sentirnos tentados de añadir que esa retirada ha sido paralela al progreso de la idea misma de una búsqueda estrictamente racional de la verdad, y del desarrollo de la ciencia moderna (de Galileo a Newton). Pues aunque la teología es obra de la razón, se apoya sobre la fe en la Revelación y está sujeta al control del guardián de esa fe, que para los católicos es el Magisterio de la Iglesia. Cabe recurrir a San Agustín para justificar esta tesis, a partir de su distinción entre el saber en sentido propio -la ciencia-, que debemos a la razón, y la fe, que depende de la autoridad (de las Escrituras, del profeta o de sus portavo- 
ces): «quod scimus debemus rationi, quod credimus auctoritati» (De util. cred. IX, 25). Por otra parte, a la revolución científica hay que sumarle el aumento de la increencia y la difusión de una organización política basada en la neutralidad religiosa. El ideal de la ciencia y de la política moderna, el ideal de la Ilustración (sapere aude, salir del estado de infancia para llegar a la autonomía), lleva de este modo a expulsar fuera del campo de la razón, y por lo tanto, fuera de la universidad, cualquier dependencia respecto de una autoridad.

Desde ese punto de vista, Francia representa sin duda un caso extremo: tras la abolición de la universidad por la Revolución, la Tercera República suprimió el derecho a otorgar títulos -sobre todo el Doctorado- a las universidades privadas, y de paso, la propia denominación de universidad, estableciendo un monopolio estatal sobre la institución universitaria y dejando a la Iglesia católica la posibilidad de mantener «institutos católicos», que eventualmente podrían tener el estatuto de universidad pontificia, pero sin reconocimiento por parte del Estado francés. Al mismo tiempo, las facultades de teología fueron eliminadas de la universidad, de manera que hoy en día la teología está ausente del mundo universitario en sentido estricto, con excepción de los departamentos de Alsacia-Mosela, que en aquel momento pertenecían a Alemania y desde 1918 se mantienen sujetos al régimen del Concordato, no al de la separación de Iglesia y Estado (leyes de 1905) ${ }^{1}$. Sin embargo, esta radical evolución institucional se corresponde, a mi parecer, con una evolución bastante generalizada en Occidente, incluso en los países en los que están reconocidas las universidades confesionales (Italia, España) y en aquellos en los que las universidades públicas hacen hueco a la enseñanza de la teología (las Divinity Schools en Estados Unidos).

Está bastante extendida la idea de que la autoridad no se lleva bien con la ciencia y de que la teología depende de la autoridad, no sólo en cuanto a sus premisas, sostenidas por la fe, sino también en cuanto a sus conclusiones, que están sujetas a un control del Magisterio. Este dominio de la autoridad también es discutido a veces incluso por los propios teólogos, que reivindican una «autonomía» legítima frente a la autoridad eclesiástica, de modo semejante a otros saberes y disciplinas. Aunque se esté dispuesto a admitir que la teología encuentra el punto de partida de su reflexión de una manera muy particular y

\footnotetext{
${ }^{1}$ Acerca de este asunto, véase la comunicación del P. GARRIGUES, J.-M., al Senado francés en 2009, «Quelle place pour la théologie à l'université», que se puede escuchar online en: http://www.canalacademie.com/ida4097-Quelle-place-pour-la-theologie-a-l-Universite.html.
} 
diferente a la de la experiencia común, a partir de un texto sagrado, se rechaza que la investigación teológica deba estar sometida a un control que no sea el de la razón y el de la comunidad de expertos. Es fácil comprender que las facultades de teología protestante están menos expuestas que las facultades católicas a esa impugnación que procede tanto del exterior como del interior, dado que no hay autoridad comparable al magisterio católico que guíe, controle, confirme o condene el trabajo de los teólogos.

No deseo adentrarme demasiado en un terreno tan complejo. Estas pocas consideraciones sólo pretenden poner en evidencia que la cuestión de la racionalidad y de su relación con la autoridad se encuentra en el núcleo del proceso de marginación, e incluso desaparición de la teología en las universidades, y atañe a la naturaleza misma de la teología y de la universidad.

Para tratar de aportar algunos elementos de reflexión constructivos, me gustaría exponer brevemente la importante idea que desarrolla el papa Benedicto XVI sobre la necesidad de una racionalidad ampliada, y después extraeré algunas consecuencias de ella.

\section{LA IDEA DE RACIONALIDAD AMPLIADA}

Aunque nunca ha publicado un texto dedicado de forma explícita a la universidad, Benedicto XVI se ha expresado sobre este tema con frecuencia, en particular en distintos discursos dirigidos parcial o exclusivamente a la comunidad universitaria ${ }^{2}$. De hecho, cabe señalar que este papa ha privilegiado un tipo de expresión relativamente nuevo: la «lección universitaria» (como Pío XII utilizó el mensaje radiofónico, Juan Pablo II la encíclica y Francisco actualmente la homilía) ${ }^{3}$. Como Prefecto de la Congregación para la Doctrina

${ }^{2}$ El P. IDE, P., esboza una síntesis de las intervenciones más importantes de Benedicto XVI acerca de la universidad y la enseñanza de la teología, en «L'idée d'Université selon Benoît XVI», Seminarium 50 (2010/4) 765-799. Además de las tres conferencias que se mencionan en la siguiente nota, cabe destacar los siguientes discursos: con ocasión de la inauguración del año académico en la Universidad Católica del Sagrado Corazón (Milán, 25 de noviembre de 2005); a los participantes en el Encuentro europeo de profesores de universidad (Roma, 23 de junio de 2007); a los educadores católicos, en la Universidad Católica de América (Washington, 17 de abril de 2008); a los participantes en el sexto Simposio europeo de profesores universitarios (Roma, 7 de junio de 2008); a los profesores y alumnos de las universidades católicas romanas (Roma, 19 de noviembre de 2009). Todos ellos se pueden consultar en la página www.vatican.va.

3 En particular, la conferencia impartida en la Universidad de Ratisbona («Fe, Razón y Universidad: recuerdos y reflexiones») el 12 de septiembre de 2006; la conferencia prevista para la Universidad de La Sapienza («Mantener despierta la sensibilidad a la verdad») el 17 de enero de 
de la Fe ya había practicado en numerosas ocasiones este ejercicio, que daba continuidad a sus enseñanzas como profesor. Así sucedió en Francia en una serie de conferencias sobre la catequesis en los años 80, o en una conferencia en la Sorbona la víspera del año 2000, con ocasión de un coloquio acerca de la situación actual del cristianismo ${ }^{4}$.

Desconozco el papel que pudo tener el Cardenal Ratzinger en la importante exhortación apostólica de Juan Pablo II acerca de las universidades católicas, Ex Corde Ecclesiae. Sin embargo me parece de interés recordar, como preámbulo, los fines que ese texto atribuye a la universidad católica, que son, al menos los tres primeros, objetivos válidos para todas las universidades en general. Se trata de: 1) la búsqueda de la verdad, 2) su transmisión, 3) la integración de los saberes y 4) el diálogo entre la razón y la fe, junto con la promoción de la teología $a^{5}$ Se aprecia que las dos primeras tareas son la investigación y la enseñanza, sin privilegiar una sobre otra: la universidad es a la vez laboratorio y escuela, lugar de enseñanza de la ciencia por parte de los mismos que elaboran la ciencia. Las dos contribuyen a la «diaconía de la verdad», que es la misión de la Iglesia.

En varias ocasiones, Benedicto XVI se ha manifestado acerca de la crisis de la universidad, ofreciendo un análisis que parece vinculado en gran medida a esta cuádruple misión. Subraya igualmente cuatro factores en esa crisis: 1) la disgregación de las disciplinas o la fragmentación del conocimiento en especialidades y subespecialidades, sin apenas comunicación entre ellas, y sin un punto de vista superior, o dedicado a las cuestiones más fundamentales relativas al ser humano y la sociedad; 2) la reducción instrumental de la enseñanza universitaria, sujeta cada vez más a exigencias de utilidad, o incluso del mercado, que someten la institución a criterios económicos; 3) el escepticismo, que ya no permite plantear la pregunta acerca de la verdad fuera de las disciplinas empíricas y/o matemáticas, limitándose así al conocimiento positivo; 4) la secularización, paralela a la de la sociedad, que lleva a expulsar la cuestión de Dios fuera de los muros de la universidad y, con ella, el estudio científico

\footnotetext{
2008; y la conferencia destinada al mundo de la cultura en el Colegio de los Bernardinos de París, el 12 de septiembre de 2008 (sobre «Los orígenes de la teología occidental y las raíces de la cultura europea»).

${ }^{4}$ RatzingeR, J., «Vérité du Christianisme». Se impartió en el coloquio «2000 ans après quoi?», editado por Michon, C., Christianisme. Héritages et Destins, Paris: Le livre de poche, 2002.

${ }^{5}$ Juan Pablo II, Constitución Apostólica Ex Corde Ecclesiae, sobre la universidad católica, Vaticano, 1990.
} 
de la Revelación o de los grandes textos teológicos de la tradición occidental cristiana.

He recordado estos elementos de forma desordenada y es legítimo tratar de encontrar relaciones de causa y efecto. La cuestión no es sencilla: ¿el estallido de las disciplinas se debe a su desarrollo interno, que fomentaría de forma inevitable la especialización y por lo tanto la fragmentación, o se deriva del escepticismo respecto a la idea de una unidad y una función universal del conocimiento, o incluso de la naturaleza del ser humano? ¿Es el ateísmo la consecuencia de un escepticismo generalizado acerca de las grandes cuestiones de la humanidad, o es la causa del mismo? Parece bastante claro, a partir de las distintas intervenciones de Benedicto XVI sobre el asunto, que él considera el positivismo utilitarista y la fragmentación del conocimiento como consecuencias del escepticismo respecto de la capacidad de la razón humana para afrontar las cuestiones metafísicas, y que ese escepticismo es, a su vez, fruto de la secularización del espíritu. Esto provoca una evolución histórica que parte del escepticismo producido por la secularización de los Tiempos Modernos y de la Ilustración; ese escepticismo estuvo frenado por el dominio del pensamiento marxista hasta los años 60 , al precio de un positivismo de combate que condujo -tras la crisis ideológica del marxismo- al liberalismo actual, que a su vez consagra la fragmentación de las disciplinas y el enfoque utilitarista y mercantil de la enseñanza superior.

Este diagnóstico, inevitablemente esquemático, no es completamente original, y muchos de sus elementos son aceptados por todo el mundo, aunque no se evalúen de la misma forma. Más significativo es el remedio propuesto por Benedicto XVI contra esta crisis de la universidad, que es en gran medida, aunque no solamente, una crisis de las humanidades y, por lo tanto, una crisis de la humanidad. Se contiene en un concepto con diversas ramificaciones: el de la razón o la racionalidad ampliada. A través de él se plantea que la crisis se debería a una reducción del pensamiento humano, consecuencia y factor de progresos parciales innegables (desarrollo de la ciencia moderna, y de la o de las técnicas), pero cuyo coste es a la vez una pérdida de sentido y de valor. Se pueden distinguir con Pascal Ide, de quien tomo prestados algunos de estos análisis, tres ampliaciones de la racionalidad promovidas por Benedicto XVI ${ }^{6}$.

${ }^{6}$ IDE, P., «L'idée d'université selon Benoît XVI», o.c. 
1. La primera es la ampliación de la ciencia a la sabiduría. En un «Discurso a los participantes en el Encuentro europeo de profesores de universidad» (Roma, 23 de junio de 2007), el Papa decía: «La universidad, por su parte, jamás debe perder de vista su vocación particular a ser una "universitas", en la que las diversas disciplinas, cada una a su modo, se vean como parte de un unum más grande. ¡Cuán urgente es la necesidad de redescubrir la unidad del saber y oponerse a la tendencia a la fragmentación y a la falta de comunicación que se da con demasiada frecuencia en nuestros centros educativos!». En una línea que recuerda al Husserl de la Krisis, veía en esa preocupación por la unidad, que podemos calificar arquitectónica (pensando en Aristóteles y en la idea de que «es propio del sabio ordenar» ${ }^{7}$ ), una marca de la identidad europea: $\ll$ El esfuerzo por reconciliar el impulso a la especialización con la necesidad de preservar la unidad del saber puede estimular el crecimiento de la unidad europea y ayudar al continente a redescubrir su "vocación" cultural específica en el mundo de hoy» ${ }^{8}$. Un enfoque verdaderamente universal debe tener en cuenta no sólo las ciencias positivas, sino también los distintos recursos de la cultura, el arte, la literatura, la religión y, en particular, la teología. Esto nos lleva a la segunda ampliación de la razón:

2. La ampliación de la razón a la fe. Sin llegar a hacer una apología de la teología natural, Benedicto XVI ha defendido en muchas ocasiones el tema tradicional de la concordancia de la fe y la razón, de la pertenencia del cristianismo a la «teología natural de los filósofos», más que a la teología mítica de los poetas o a la teología civil de las naciones. También ha insistido en el carácter racional del Dios de la Biblia, en el carácter providencial de la helenización de la revelación cristiana y en la concordancia fundamental de los mandamientos de Dios con los de la Razón`. Ha hablado igualmente de la «purificación recíproca» de la razón por la fe y de la religión por la razón, denunciando conjuntamente las patologías del racionalismo y las del fundamentalismo religioso. Se ha planteado también, siguiendo a Juan Pablo $\mathrm{II}^{10}$, la le-

\footnotetext{
${ }^{7}$ ARISTÓTELES, Metafísica I, 982a17-18.

${ }^{8}$ BENEDICTO XVI, «Discurso a los participantes en el Encuentro europeo de profesores de universidad» (Roma, 23 de junio de 2007). Esta exigencia se hace eco también de un tema desarrollado por Urs von Balthasar acerca del carácter «sinfónico» de la verdad. Véase VON BALTHASAR, H. U., La vérité est symphonique. Essai sur le pluralisme chrétien, Paris: Parole et Silence, 2000.

9 Es un aspecto importante de la conferencia en Ratisbona: BENEDICTO XVI, «Fe, Razón y Universidad: recuerdos y reflexiones», 12 de septiembre de 2006.

${ }^{10}$ Juan Pablo II, Encíclica Fides et Ratio, 14 de septiembre de 1998.
} 
gítima autonomía de la investigación científica y la tensión que podría existir, para el teólogo, entre la libertad académica de la ciencia y el reconocimiento de la autoridad del Magisterio. Sin abordar todavía ese espinoso asunto, podemos subrayar ya la idea de que la fe cristiana, y tal vez también los contenidos metafísicos o morales de las religiones del mundo, excluidos de la ciencia positiva, no deberán quedar excluidos de la racionalidad y de su despliegue dentro de la universidad. Es más, el punto de vista teológico es un punto de vista arquitectónico, un punto de vista superior, que busca la unidad del saber, y su orientación, ya que considera a Dios y a las demás realidades en cuanto se refieren a Dios ${ }^{11}$. Ahora bien, al considerar un Dios que es a la vez Razón y Amor, el punto de vista teológico lleva a una tercera ampliación de la racionalidad.

3. La ampliación de la comprensión intelectual a la acción ética, incluso al amor fraternal. En su «Discurso a los educadores católicos», en la Universidad Católica de América (Washington, 17 de abril de 2008), Benedicto XVI se expresaba de la siguiente forma: «Verdad significa más que conocimiento: conocer la verdad nos lleva a descubrir el bien. La verdad se dirige al individuo en su totalidad, invitándonos a responder con todo nuestro ser». Esta inquietud por la traducción en actos, o incluso por una expresión «performativa» de la $\mathrm{fe}^{12}$, es también válida para la actividad de investigación y enseñanza de la universidad. Es más, la preocupación por no limitar la función de la universidad a la elaboración y transmisión de conocimientos, sino por hacer de ella un laboratorio y una escuela de «antropología concreta», que remita al ser humano en su totalidad, permitiría responder al desafío contemporáneo de la Universidad como preparación para la vida profesional. Veo en esta propuesta de una inquietud ética, incluso de un ejercicio de la caridad cristiana en todos los niveles, entre profesores, entre alumnos, entre unos y otros, y de todos con el personal administrativo, el indicio de que el remedio a la crisis no se presenta como una vuelta atrás (recuperar la universidad original), sino como un desafío para el futuro, un desarrollo -el de la dimensión ética- que las circunstancias actuales (masificación, profesionalización) hacen sin duda más urgente que en el pasado.

11 Tomás de Aquino, Suma contra los Gentiles, libro II, cap. 4.

12 Encíclica Spe Salvi del 30 de noviembre de 2007, que introduce el concepto en el n. 2 y posteriormente lo retoma. 


\section{3. ¿ENSEÑAR TEOLOGÍA EN UNA UNIVERSIDAD SECULAR?}

Bastantes de las consideraciones anteriores pueden lograr un amplio consenso, tanto en cuanto al diagnóstico como al remedio. En todo caso, no remiten a una visión confesional y se basan en hechos y análisis racionales.

Se comparte lo que afirma Benedicto XVI acerca de la primacía de la economía y la política. La masificación y la presión para que la universidad se focalice en la preparación de futuros profesionales, son dos características de las universidades europeas que no están exentas de problemas. De modo semejante, hay acuerdo en torno al deseo antes formulado de una formación más integral, dirigida al ser humano en su totalidad, en la que se reúnan la formación de la voluntad con la del intelecto y la acción con la teoría en la preocupación por los demás, en particular por los más desvalidos. Ese deseo encuentra un verdadero eco en nuestros contemporáneos, y en particular en los jóvenes, los primeros afectados. El desarrollo de actividades «para-universitarias» de índole social, incluso humanitaria, así como el aumento de intercambios internacionales que se orientan más al enriquecimiento cultural que a obtener un resultado inmediato, son señales de este fenómeno. Y pienso que sucede algo parecido con la insistencia en el papel del testimonio personal de los profesores, primer vector de la diaconía de la verdad que debe caracterizar la universidad, así como en la dimensión ética propia de la ciencia, o de forma más general, propia de la vida común de una institución tan completa como una universidad. Es más, para un cristiano preocupado por dar testimonio esta invitación es pertinente, ya que el testimonio en cuestión va más allá de una forma de apostolado del discurso público, que no se aceptaría en el seno de una institución laica, y se traduce en el del ejemplo a través de la seriedad profesional y la consideración hacia las demás personas.

Llego por tanto al punto que más nos afecta: el lugar que ocupa la teología desde el punto de vista de la racionalidad abierta. Considero que hay que tener en cuenta dos aspectos: por una parte, la necesidad de sabiduría, que ninguna ciencia en particular podría asumir precisamente debido a la fragmentación de los saberes; por otra parte, la cuestión de la legítima pertenencia de la teología a la enseñanza universitaria, en nombre de su racionalidad.

No estoy convencido de que hoy en día pueda haber una ciencia arquitectónica, como pensaba Aristóteles de la metafísica y Santo Tomás de la 
teología ${ }^{13}$. Y si existe una reflexión acerca de los principios de las ciencias, corresponde más a la filosofía que a la teología. Dicho esto, aunque ya no tiene cabida la idea de una arqui-ciencia que fundara a todas las demás y les diera su razón de ser, sigue existiendo la demanda de un sentido para la actividad científica y su enseñanza y, por lo tanto, para la universidad. El hecho de la fragmentación del saber y la multiplicidad de las concepciones y visiones del mundo indican la necesidad de disponer de espacio para un pensamiento más fundamental, que permita al menos una base común de reflexión para todos los actores de la investigación y de la enseñanza. En ocasiones se favorece la interdisciplinariedad pero, aunque se consiguen algunos logros, a menudo se trata más de discursos que de realidades, y los contactos entre distintas disciplinas terminan siendo episódicos. Algunas instituciones mantienen una formación común inicial, o bien ofrecen cursos transversales de filosofía, antropología o incluso de teología. Pero son excepciones. E incluso en estos casos, no cabría decir que esa formación humanista, extendida a todas las generaciones de alumnos, se corresponda con lo que fue la organización de la universidad medieval y moderna. No obstante indica que, a día de hoy, la teología todavía puede contribuir a ese papel de sabiduría.

Sin embargo, conviene subrayar que cuando la teología ha encontrado derecho de ciudadanía en la universidad, con frecuencia ha evolucionado como una disciplina universitaria más, no sólo a causa de los exámenes, grados y diplomas, sino también por la especialización, los avances internos, los debates entre iguales, etc., que le han retirado su función arquitectónica inicial. Y sobre todo, como ya se ha indicado, ahora no puede beneficiarse de la unanimidad de la fe que existía entre los escolásticos medievales, y en consecuencia, se ve relegada a la particularidad de una creencia religiosa cuyos seguidores a menudo no son mayoría. ¿Cómo atribuirle entonces un lugar en la universidad secular?

Esbozo a continuación una respuesta a esta pregunta con algunas breves consideraciones.

En primer lugar, partiendo del caso extremo francés, si la visión secular del saber y de la universidad excluye la enseñanza de una teología que po-

13 Véase Tomás de Aquino, Suma contra los Gentiles I, c. 1, que coteja las citas de Aristóteles y de las Escrituras para ilustrar la función ordenadora de la sabiduría; y Suma teológica, I, q. 1. 
dríamos llamar «militante», no excluye la de las doctrinas teológicas. Las capellanías deben situarse fuera de la universidad, pero no está prohibido enseñar los contenidos doctrinales del cristianismo (o del islam) en los anfiteatros. En Francia, donde toda la universidad es secular y la teología no tiene cabida, han empezado a oírse desde hace dos o tres décadas voces muy «laicas» que reclaman que se enseñe en la escuela el «hecho religioso». Se percibe en esa aspiración una especie de necesidad política (saber quién es quién y quién piensa qué), o simplemente cultural. La ignorancia religiosa hace inaccesibles e incomprensibles las obras maestras de la literatura clásica (Montaigne, Pascal, Racine e incluso Voltaire o Víctor Hugo, por limitarme a las francesas, pero habría que añadir a Dante, Cervantes, Shakespeare o Dostoyevski) y en mayor medida las de la pintura y la arquitectura. Un director de la Escuela de Bellas Artes se lamentaba hace algunos años de que una alumna, tras cinco años de escuela superior, pudiera preguntar delante de un Rafael quién era el hombre con alas que estaba ante la joven... La enseñanza del «hecho religioso» planteada con el fin de paliar un llamativo déficit de cultura, no podría darse en la universidad sin un interés por profundizar en las especulaciones teológicas que dieron forma, por ejemplo, a la constitución de la doctrina cristiana y que aún hoy desempeñan un papel importante. De este modo, a pesar de una hostilidad en ocasiones vehemente y de los estrictos principios que organizan el espacio público, se abre paso una cierta demanda de conocimientos teológicos. Con frecuencia, esta demanda se ve parcialmente satisfecha por los profesores de las disciplinas afectadas: filosofía, historia e historia del arte, literatura, ciencias políticas y derecho.

Segundo punto. No se trata únicamente de un interés positivo por lo que dicen y piensan los eruditos de una tradición religiosa: el discurso teológico es relevante para la cultura contemporánea, ya que es una fuente importante de reflexión sobre el hombre, la sociedad, la cultura y los distintos saberes. Ignorar las aportaciones de Karl Barth o Karl Rahner, de Henri de Lubac, de Urs von Balthasar, de Jurgen Moltmann o de Joseph Ratzinger constituye una privación para el pensamiento. El éxito editorial de algunas encíclicas de Juan Pablo II, como en la actualidad las del papa Francisco, manifiestan un interés que va más allá del círculo de los fieles, y sería absurdo ignorarlo. En cuanto a Benedicto XVI, sus diálogos directos o indirectos con Habermas o Giorgio Agaben o Paolo Flores d'Arcais hacen de él un verdadero interlocutor y protagonista del pensamiento contemporáneo. La invocación que hace Benedicto XVI 
de una racionalidad «abierta» afecta a toda forma de pensamiento y de sabiduría irreductible a las ciencias de la naturaleza, e incluso a las ciencias humanas cuando se desarrollan según el modelo de las ciencias empíricas. La filosofía, el arte, la/o las religiones y, por tanto, la/o las teologías pertenecen a la razón ampliada, y son portadoras de un sentido que las ciencias empíricas no pueden ofrecer. Es más, la historia de la teología cristiana está en parte vinculada al desarrollo de la ciencia moderna. Por discutibles que puedan ser las obras de P. Duhem y Amos Funkenstein, ponen en evidencia la conexión de la teología bíblica y de la ciencia positiva en la historia de la racionalidad occidental.

Este punto me lleva a una tercera consideración. La racionalidad teológica no es fundamentalmente diferente de la de la filosofía y las ciencias y, como éstas, no desemboca en el abandono de la verdad ni en el relativismo. Esto choca con el espíritu de los tiempos, que reconoce una especie de verdad universal en el ámbito de las ciencias mientras, por el contrario, defiende el relativismo de los valores (morales) y de las creencias religiosas. El ethos del Cristianismo también es antirrelativista en relación con los valores y pone la verdad como sustento de la práctica (y no a la inversa). En esto, la fe (y por tanto la teología) se muestra más optimista respecto a la razón humana que la propia razón, al menos hoy en día. Éste era uno de los temas de la famosa encíclica Fides et Ratio. La fe pueda aportar a la razón la audacia, la parrhesia, que a veces parece faltarle. De una forma u otra, la presencia de la enseñanza de la teología e incluso de una investigación teológica en la universidad supone dar la bienvenida a un discurso racional, una verdadera obra de razón científica y una contribución a la constitución de los saberes y de la cultura humana.

En efecto, hay que distinguir, con Tomás de Aquino ${ }^{14}$, los debates teológicos que tienen por fin establecer lo que se debe creer para uso de los fieles, la «verdad teológica» por llamarlo así, el an ita sit; y aquellos cuyo objetivo es explicar y comprender esas tesis, proponiendo argumentos, un intellectus fidei que responda a la cuestión de por qué y cómo es así, cur et quommodo ita sit. En el primer tipo de discusión, dice Tomás, todo es útil para establecer la verdad: la razón que compartimos con los paganos, la autoridad de las Escrituras que compartimos con los judíos, para el Antiguo Testamento, y con los cristianos,

${ }^{14}$ Tomás DE Aquino, Quodlibet IV, q. 9. 
para el Nuevo, e incluso la autoridad del Magisterio cuando la discusión se plantea entre católicos. Pero si el auditorio ya está de acuerdo acerca de las verdades que se deben creer, el uso de las autoridades sería vano y los alumnos se marcharían «con la cabeza vacía» (vacuus abscedet). Únicamente haciendo uso de la razón, de los argumentos sometidos a la evaluación imparcial y universal de esa facultad común, es como progresarán en la comprensión de lo que ya creen. La teología así entendida consiste menos en saber que en comprender y eso la aproxima curiosamente a la filosofía ${ }^{15}$. Esta comprensión de las razones de la fe es accesible también al no creyente ${ }^{16}$.

¿Pero qué enseñanza de la teología puede darse en una universidad secular, en la que la mayoría de sus miembros (profesores y alumnos) no comparten la fe religiosa? La teología católica, estudio de la fe cristiana para uso de los fieles, se somete al Magisterio en sus principios y en la publicación de sus conclusiones, se ejerce «en la fe», va «de la fe a la fe», como diría K. Barth. ¿Cómo podría desarrollarse en el marco de una universidad secular?

Considero que la inclusión de una enseñanza de la teología en una universidad secular puede hacerse de dos formas, según que se confíe o no a la autoridad religiosa la tarea de cualificar a los profesores y los programas. En el primer caso, hay una autoridad, externa a la universidad, que se ejerce sobre una disciplina y sus profesores. Esta situación, aceptable desde el punto de vista de la autoridad religiosa (Iglesia), no está exenta de tensiones y puede marginar a la teología, reservándola a los creyentes y a algunos curiosos. Bajo esta forma la teología puede estar presente en la universidad y gozar de un estatus de disciplina universitaria, pero su situación es inestable, constituye un encla-

${ }^{15}$ He desarrollado parcialmente estas ideas, a propósito de Tomás de Aquino, en «Philosophie de haut en bas. La théologie (scolastique) comme philosophie (analytique) de la foi (chrétienne)», Revue internationale de philosophie 225 (2003/3) 229-249.

${ }^{16}$ Tomás de Aquino consideraba que la teología es una ciencia a partir de un concepto de «ciencia» muy exigente que tomaba de Aristóteles: la ciencia produce demostraciones ciertas a partir de premisas evidentes, conocidas por uno mismo. Ciertamente, en esta vida el teólogo posee estas premisas por la fe, y por tanto sin evidencia, pero los bienaventurados sí las conocen con evidencia. «Nuestra teología» puede considerarse una ciencia subordinada a la teología de los bienaventurados, igual que la música se subordina a la matemática, que le aporta sus principios: los principios de la música no son evidentes para el músico, pero sí para el matemático. Podemos considerar, con Guillermo de Ockham, que se trata de un subterfugio y que la teología no es una ciencia para nosotros. Sin embargo, el movimiento racional que va de las premisas a las conclusiones tiene para Tomás de Aquino la apariencia de ciencia. Esta apparentia scientiae es completamente racional, incluso aunque no se trate de puras deducciones silogísticas. Los razonamientos, distinciones y argumentos pueden ser comprendidos por todos, creyentes y no creyentes. 
ve, como una capellanía erudita. En el segundo caso, la teología es considerada una fuente para la reflexión, en el marco de una racionalidad ampliada, y no se corresponde necesariamente con la teología de los eruditos de una tradición religiosa, que tratan de profundizar en su tradición, encontrar razones y alimentar la comprensión que tienen los fieles del contenido de su fe, o de la Revelación. Un no creyente, o un creyente muy poco ortodoxo, es capaz de entender y exponer las «razones de la fe» (rationes fidei). Semejante teología «laica» o en estado «salvaje» o «extramuros» no está necesariamente en conflicto con la teología de la Iglesia, y evidentemente puede ser desarrollada por un teólogo creyente, incluso sometido personalmente a la autoridad religiosa. Como presentación pedagógica y no confesional de la teología clásica (interna), o verdadera especulación llevada a cabo fuera del control de la autoridad, puede hacer una aportación efectiva (como la de un think tank teológico). Esta puerta abierta podría ofrecer también un elemento de solución para los teólogos que experimentan un conflicto entre la práctica autónoma de su disciplina y la autoridad de la que dependen: de este modo, tendrían la posibilidad de expresar sus opiniones sin control, pero también sin la pretensión de ostentar la etiqueta de «católico». No es más que una idea, sin duda criticable, pero cuya validez habría que ponderar.

Por supuesto, veo que esta idea puede plantear problemas desde el punto de vista de la Iglesia, o de la fe. ¿No cabe el riesgo de desfigurar la doctrina en las mentes de los estudiantes, por parte de ignorantes, o peor, de ideólogos opuestos a la fe? Sí, existe esa posibilidad. Pero la ignorancia también es un peligro. El desafío es que los creyentes obtengan la mejor cualificación en el conocimiento teológico, lo que no parece algo insuperable. Y finalmente, ese riesgo puede ser un reto para la parrbesia: ¿quién sabe si la fuerza de la doctrina cristiana, por ejemplo, no puede pasar a través de un discurso adverso pero competente? Es prueba de madurez exponerse a ese peligro, sabiendo que la universidad pública, no siendo un organismo religioso, no puede pretender asumir el papel de los obispos o de los maestros en la fe.

\section{ConCLUSIÓN}

Estas observaciones no han pretendido abarcar de modo completo la cuestión de la verdad y la racionalidad tal y como pueden ser abordadas desde el punto de vista de la universidad. Pero el concepto de racionalidad ampliada se ha propuesto precisamente en función de la verdad, de la verdad de los sa- 
beres científicos y también de la verdad sobre el bien del hombre y, por consiguiente, sobre la condición humana. En esta perspectiva de aproximación a la verdad integral, por las vías de una racionalidad abierta a los discursos que no pertenecen a las ciencias, si ser ajenos a la racionalidad, es como la Universidad puede, y sin duda alguna debe, hacer espacio a la teología. Un espacio que no será el mismo en las universidades seculares y en las de inspiración cristiana. Me permito concluir citando estas palabras de san Josemaría, que han inspirado estas reflexiones: «la religión debe estar presente en la Universidad; $y$ ha de enseñarse a un nivel superior, científico, de buena teología. Una Universidad de la que la religión está ausente, es una Universidad incompleta: porque ignora una dimensión fundamental de la persona humana, que no excluye -sino que exige- las demás dimensiones. De otra parte, nadie puede violar la libertad de las conciencias: la enseñanza de la religión ha de ser libre, aunque el cristiano sabe que, si quiere ser coherente con su fe, tiene obligación grave de formarse bien en ese terreno» ${ }^{17}$.

17 S. JOSEMARÍA ESCRIVÁ, Conversaciones con Monseñor Escrivá, 73. 


\section{Bibliografía}

GARRIGUES, J.-M., «Quelle place pour la théologie à l'université», en http://www.canalacademie.com/ida4097-Quelle-place-pour-la-theologiea-l-Universite.html.

IDE, P., «L'idée d'Université selon Benoît XVI», Seminarium 50 (2010/4) 765799.

Michon, C., «Philosophie de haut en bas. La théologie (scolastique) comme philosophie (analytique) de la foi (chrétienne)», Revue internationale de philosophie 225 (2003/3) 229-249.

Ratzinger, J., «Vérité du Christianisme», en Michon, C., Christianisme. Héritages et Destins, Paris: Le livre de poche, 2002.

S. Josemaría EsCRIVÁ, Conversaciones con Monseñor Escrivá, Madrid: Rialp, 1973.

Von Balthasar, H. U., La vérité est symphonique. Essai sur le pluralisme chrétien, Paris: Parole et Silence, 2000. 